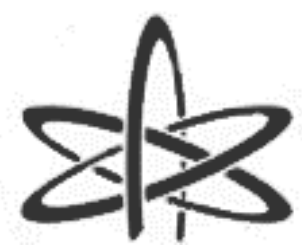

BJRS
BRAZILIAN JOURNAL

$\mathrm{OF}$

RADIATION SCIENCES

09-02B (2021) 01-16

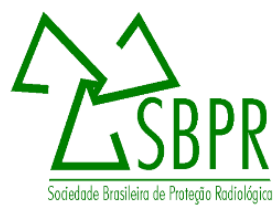

\title{
Thermal hydraulic analysis of a PWR loaded with
} annular fuel rods

\author{
Souza W.F. ${ }^{a}$, Veloso M. A. F. ${ }^{a}$, Costa A. L. ${ }^{a}$, Pereira C. ${ }^{a}$ \\ ${ }^{a}$ Departamento de Engenharia Nuclear - Escola de Engenharia \\ Universidade Federal de Minas Gerais
}

31270-901 Av. Antônio Carlos 6627, Belo Horizonte, MG

wallenfds@yahoo.com.br; mdora@nuclear.ufmg.br; antonella@nuclear.ufmg.br; claubia@nuclear.ufmg.br

\begin{abstract}
In 2006, the final report of the project named High Performance Fuel Design for Next Generation PWRs was published by the Massachusetts Institute of Technology Center for Advanced Nuclear Energy Systems. This report presented the proposal of the internally and externally cooled annular fuel to allow a substantial increase in power density while maintaining or improving safety margins. The thermal hydraulic conditions were calcu-

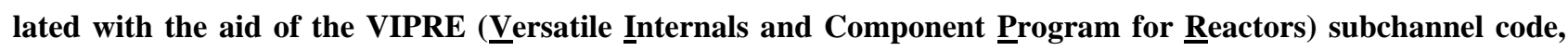
which is a widely used tool in the analysis of nuclear reactor cores. STHIPR-1 (imulação Termo-

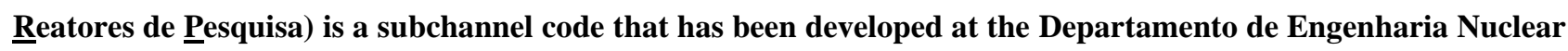
/Universidade Federal de Minas Gerais. In order to evaluate the capacity of the STHIRP program, mainly in relation to the thermal model, the new fuel concept was analyzed. The results were compared with those performed with the VIPRE code presented in the reference document.
\end{abstract}

Keywords: Annular fuel, Subchannel code, STHIRP, Thermal model

ISSN: 2319-0612

Accepted: 2021-06-01 


\section{INTRODUCTION}

The development of the annular fuel concept began in the 1950s when a technology associated with the internal and external flow of refrigerant to the fuel was implemented for the first time. [1] Since 2001, agencies and research centers worldwide coordinated by the international agencies NERI (Nuclear Energy Research Institute), MIT (Massachusetts Institute of Technology) e KAERI (Korea Atomic Energy Research Institute) have joined efforts in the process of optimizing nuclear fuels [2].

In 2006, it was published by the MIT Center for Advanced Nuclear Energy Systems, the Final Report of the Project titled High-Performance Fuel Design for Next Generation PWRs. In this report, the proposal for an internally and externally cooled annular fuel was proposed to increasing the power density (of the order of $30 \%$ or more) of a PWR reactor without prejudice to safety margins.

The transition from a solid geometry to an annular geometry has two important characteristics that allow the increase of the power density: (1) reduction of the conduction thickness, which improves from peak fuel temperature to melting, and (2) increased heat transfer surface area (in spite of a reduction of the number of fuel rods) which improves the margin of safety for Departure from the Nuclear Boiling Ratio (DNBR).

The thermal-hydraulic evaluation report published by NERI was made using the VIPRE (Versatile Internals and Component Program for Reactors) code. This program was developed for solid fuels and thus the option of calculating heat conduction in hollow tubes was used for modeling annular fuel rods. Various square array sizes were explored and the most promising options, based on DNBR considerations, were found to be the $13 \times 13$ and $12 \times 12$ arrays. The $13 \times 13$ array was selected as the most promising design.

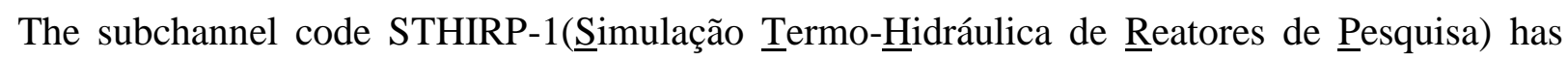
been developed by Departamento de Engenharia Nuclear da Universidade Federal de Minas Gerais (DEN-UFMG). It follows the basics principles of the subchannel technique and is intended to simulate the thermal and hydraulic phenomena that occur in the core of the water cooled research reactors under a natural convection regime. Although the STHIRP-1 code has been developed to simulate research reactors, the formulation used to describe the fluid flow and the thermal 
conduction in the fuel rods is sufficiently general to allow the application to power reactors and thermal systems that have the features for which the program was developed [3].

The increase in autonomous developemend of computational codes for the prediction of the thermal- hydraulic behavior of conventional and innovators reactors is the motivation of this work. For conventional reactors, there are many codes that simulation the solid fuel rod with great accuracy. With the advent of the new generation reactors, the evolution of the codes is necessary. Han e Chang [4] report about the difficulty in finding a code capable of simulation the internal and external cooled fuel rod which required the introduction of a simplified model in the MATRA code. In 2011 a modified model named MATRA AF has been developed for simulation of the power reactor OPR 1000 loaded with annular fuel [5].

The STHIRP code formulation allows simulation of the annular fuel without adaptations. The thermal model of the code may comprise an arbitrary number of regions which are characterized by different numbers of solid walls and distinct types of materials whose thermal properties are assumed to be a function of temperature. The first solid of the conductor does not necessarily have to be massive and it can be a fluid in motion.

In this paper the thermal model of the code was tested. The results obtained are in good agreement with those presented in the reference report.

\section{MATERIALS AND METHODS}

A typical Westinghouse 3411 MWth four loop PWR plant has been selected as the base case for the study. In the simulation, the reactor was loaded only with annular rods. The Table 1 summarizes the design data of the reference PWR core. The power adopted is $150 \%$ greater compared to nominal power of the reactor loaded with solid fuels without compromising the safety requirements of the reactor.

The analysis was developed as follows:

- Analysis of the one-rod model and comparison with the reference model.

- Analysis of the whole core model and comparison with the reference model. 
Analysis of the whole core model with power $150 \%+18 \%$ and $150 \%+18 \%$ with the change of gap heat conductance.

The operating conditions and calculation parameters are presented in the Table 1.

Table 1: Parameters of the reference reactor [6]

\begin{tabular}{ll}
\hline System pressure & $155.5 \mathrm{bar}$ \\
Fuel rod pitch & $1.651 \mathrm{~cm}$ \\
Internal diameter of fuel rods & $0.8633 \mathrm{~cm}$ \\
Guide tube outer diameter & $1.5367 \mathrm{~cm}$ \\
Fuel rod inner diameter & $1.5367 \mathrm{~cm}$ \\
Guide tube inner diameter & $1.40 \mathrm{~cm}$ \\
Fuel assembly pitch & $21.46 \mathrm{~cm}$ \\
Active fuel height & $366.0 \mathrm{~cm}$ \\
Number of heated rods per assembly & 160 \\
Chopped cosine power distribution & 1.55 \\
(ratio peak/average) & 9 \\
Number of control rods & $29.7{ }^{\circ} \mathrm{C}$ \\
Reactor thermal power & $6037.5 \mathrm{MWth}$ \\
Rate flux mass & $26.550 \mathrm{Kg} / \mathrm{s}$ \\
Inlet temperature & 27 \\
Number of channels inner & \\
\hline
\end{tabular}

The $13 \times 13$ design was chosen for the whole core modeling. Due to symmetry, the whole core model is represented by $1 / 8$ of the reactor's core. The power distribution in the hot assembly was obtained from the reference document. 
The first model consists of one fuel rod and two coolant channels - the inner channel that is inside the annular fuel rod and the outer channel that cools the outer cladding surface. (Figure1)

Figure 1: One-rod core model.

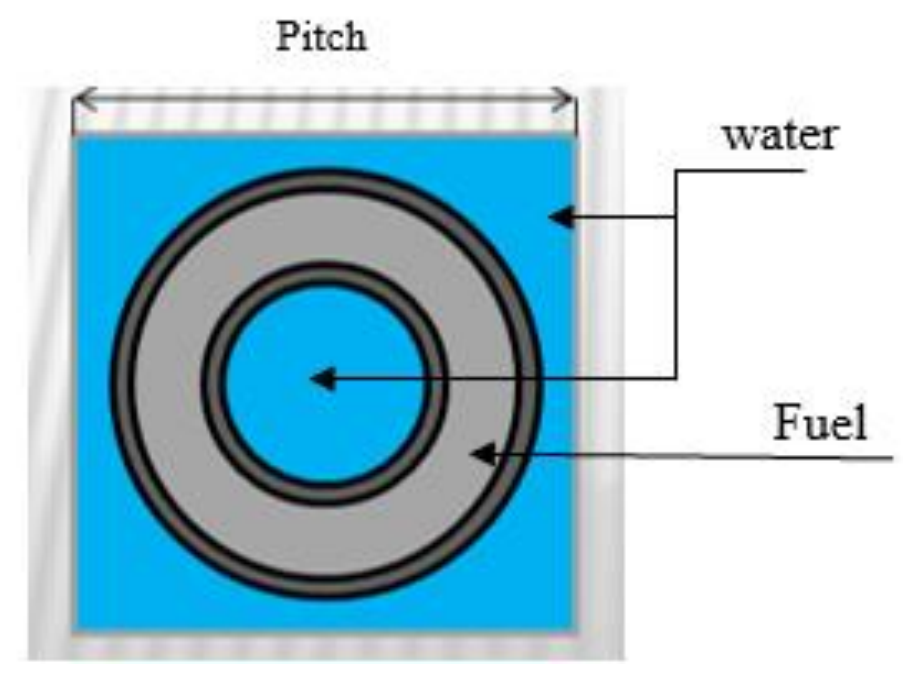

The Figure 2 shows that 5 nodal points of temperature in UO2 will be considered, in addition, 3 nodes will be considered in each cladding, one point at each end and one at the central region.

Figure 2: Pellets nodalization

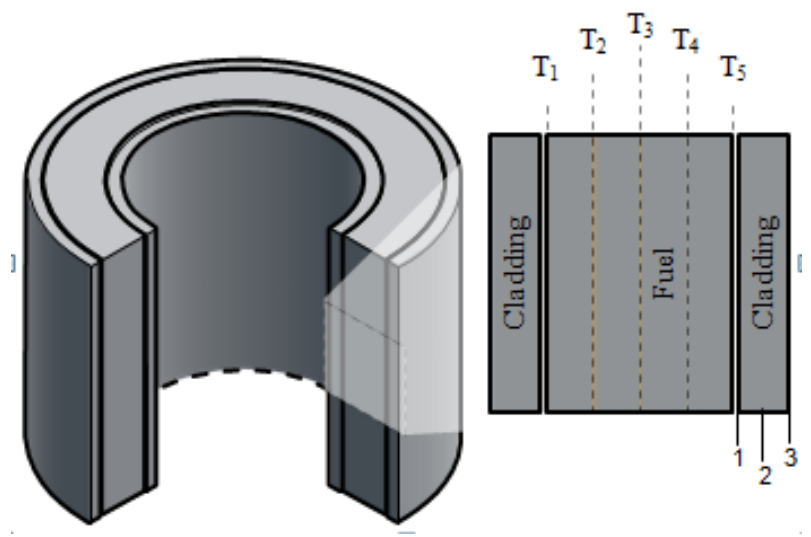

A whole core STHIRP-1 model for the 13x13 annular fuel was developed based on the power distribution and numbering schemes according to Figure 3 and Figure 4. The Figure 3 shows the 
numbering scheme next to the hot spot. The Figure 4 shows the numbering scheme of the $1 / 8$ nuclear reactor core.

Figure 3: Numbering scheme of channels and rods in the hot assembly

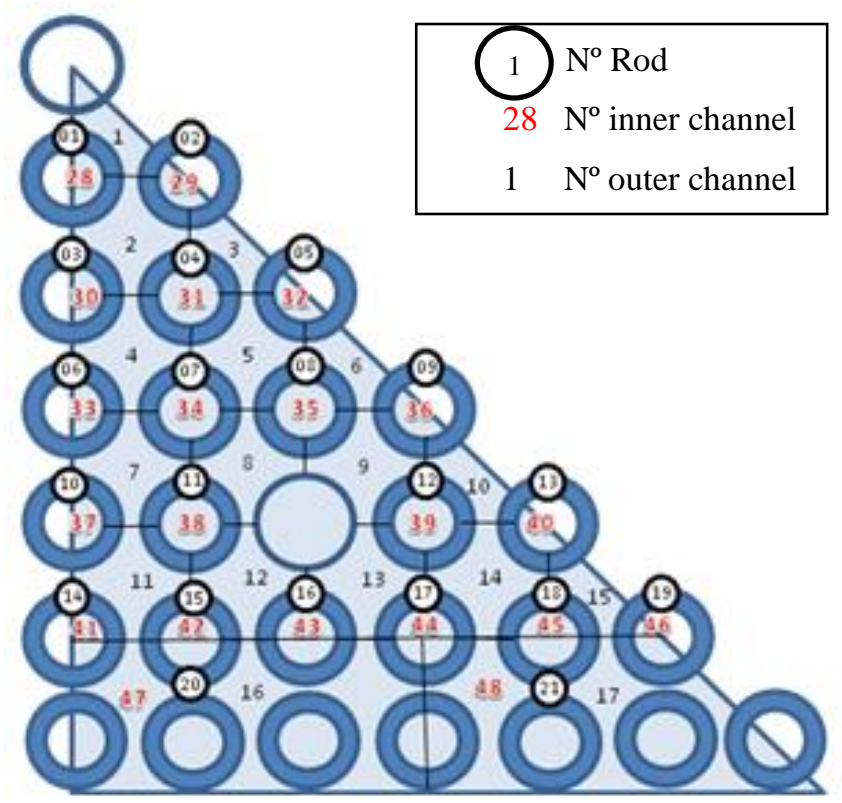

Figure 4: Numbering scheme of lumped channels and rods in $1 / 8$ core

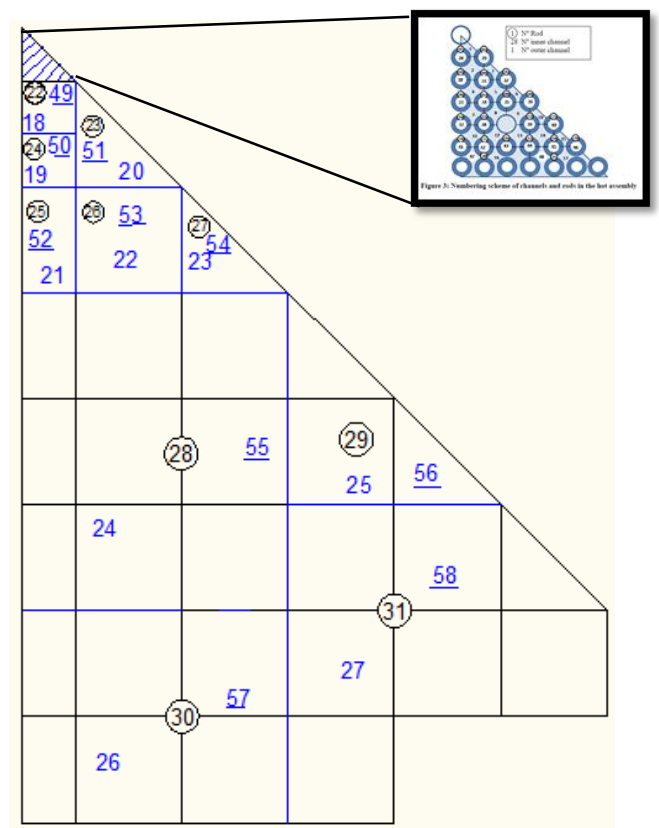

The input parameters and correlations used in the STHIRP-1 are summarized in the Table 2.

The simulation of the model was made for steady state. According to reference [5] the conductivities of external and internal gaps were considered, respectively, $\mathrm{h}_{\mathrm{go}}=1.3 * 6000 \mathrm{~W} / \mathrm{m}^{2} \mathrm{~K}$ and $\mathrm{h}_{\mathrm{gi}}=0.9 * 6000 \mathrm{~W} / \mathrm{m}^{2} \mathrm{~K}$.

The power distribution used in the reference report indicates that rod 8 is the most heated, so the analysis will be more detailed in the vicinity of that rod. The axial distribution of the heat flux was calculated from the power profile chopped cosine $\mathrm{Fz}=1.55$ [6]. The structure of the fuel element is maintained by 7 spaced grids equally spaced along the active length, with the first and the last grid located, respectively, in the axial positions $30.48 \mathrm{~cm}$ and $366.00 \mathrm{~cm}$. The conductance of the cladding- pellet gap is approximated by a constant value of $6000 \mathrm{~W} / \mathrm{m}^{2} \mathrm{~K}[6]$. 
Table 2: Input parameters and correlations for STHIRP-1[6]

Number of axial levels

41

Convergence tolerance for axial flow

0.001

Convergence tolerance for crossflow

0.01

Crossflow resistance factor, $\mathrm{K}_{0}$

0.5

Transverse momentum parameter

0.5

Turbulent mixing coefficient

0.0

Friction factor correlation

Blasius

Subcooled void correlation

Levy

Bulk void correlation

EPRI

Two-phase friction multiplier correlation

EPRI-Columbia

Critical heat flux correlation

W-3

\subsection{One-rod model}

The comparison of heat flux distribution shown in the Figure 5 is an assessment of the capacity to split heat flux in subchannels internals and externals.

Figure 5: Distribution heat flux - STHIRP and VIPRE

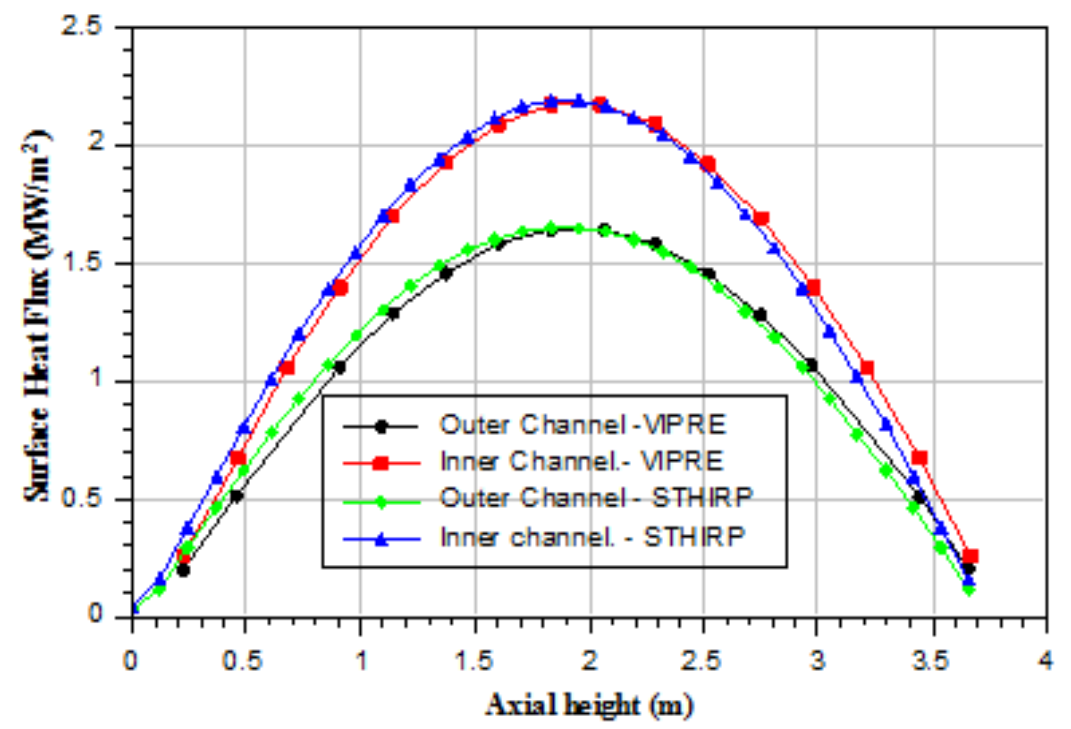


The pressure drop that occurs in the channels is presented in Figure 6. The STHIRP code mathematically adjusts the outlet pressure of the refrigerant in the channels to ensure the same pressure in all.

Figure 6: Pressure drop STHIRP and VIPRE

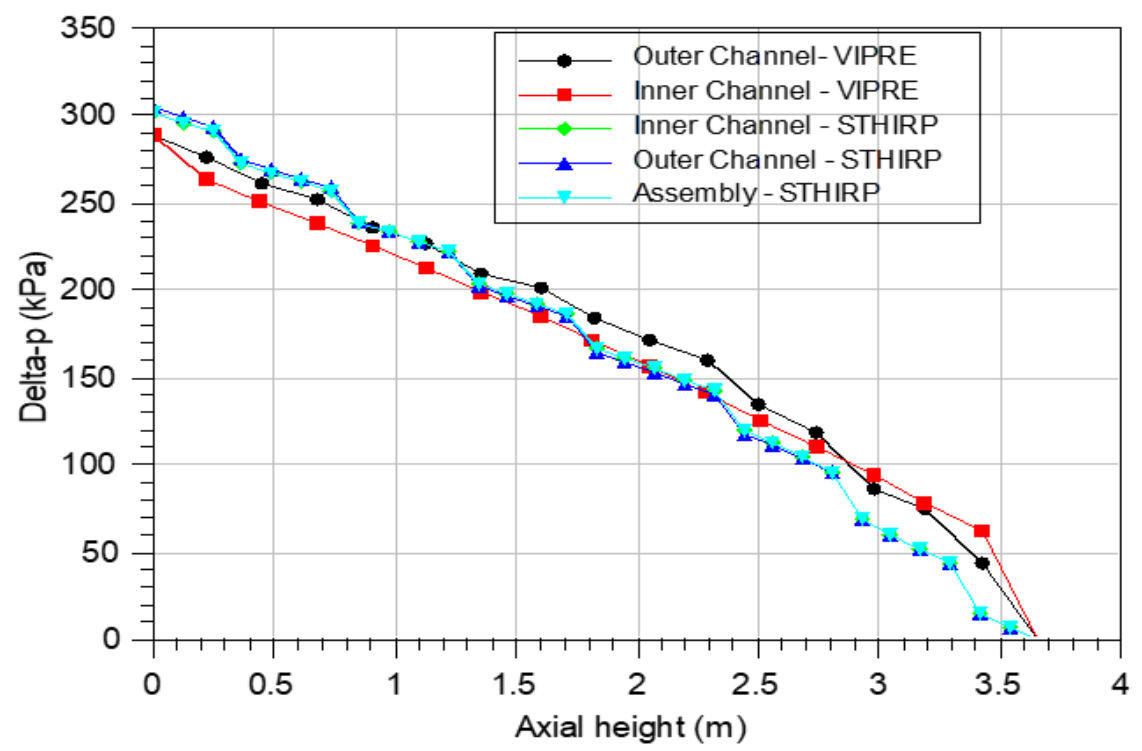

The Figure 7 shows the DNBR (Departure from Nucleate Boiling Ratio). The DNBR of the inner channel has a good agreement. The values of Minimal DNBR (MDNBR) and total pressure drop for the one-rod model are summarized in the Table 3.

Figure 7: DNBR - STHIRP and VIPRE.

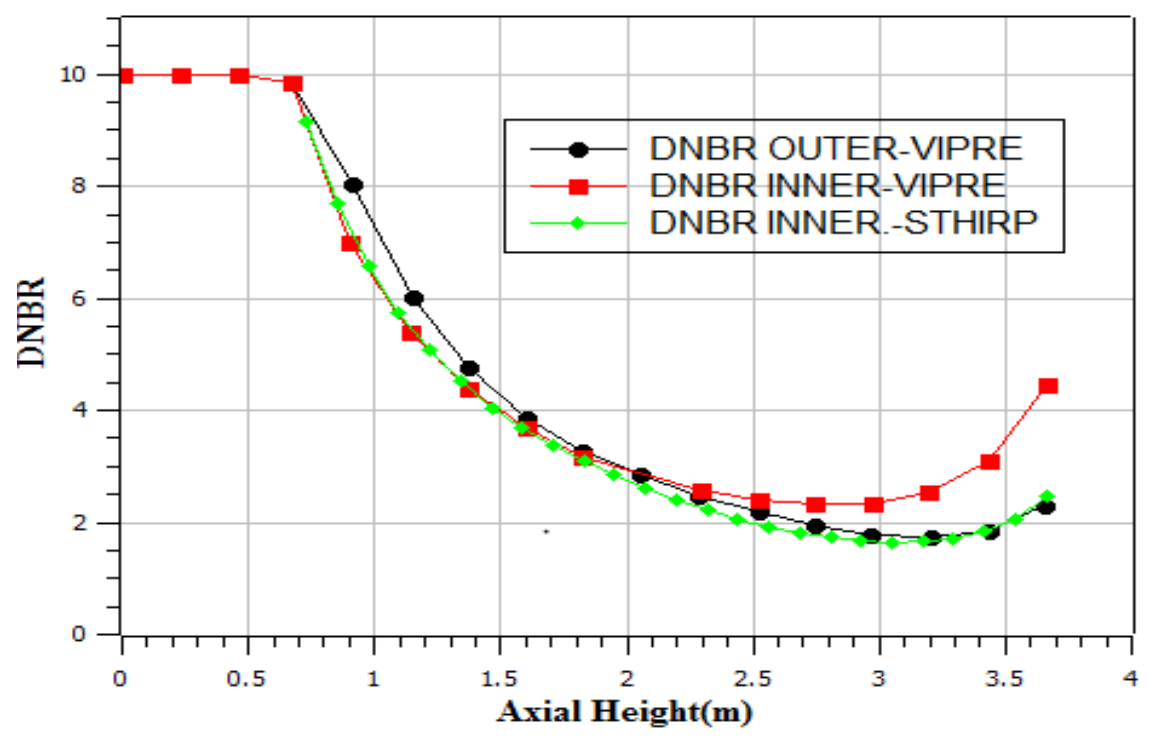


Table 3: MDNBR of the one-rod model

\begin{tabular}{ccc}
\hline & MDNBR & Total Pressure Drop (MPa) \\
\cline { 2 - 3 } One-rod model VIPRE & 1.714 & 0.288 \\
One-rod model STHIRP & 1.646 & 0.308 \\
\hline
\end{tabular}

\subsection{Whole core model - $150 \%$ Power}

Whereas that the annular fuel core has about 50\% more cooling surface than the reference solid fuel design, it can be roughly estimated that the annular fuel core can operate at $150 \%$ reference power. The results calculated with the STHIRP code for this power level are compared with those derived from the VIPRE code presented in the reference report.

Figure 8: Comparison of the heat flux distribution by whole core model and one-rod model.

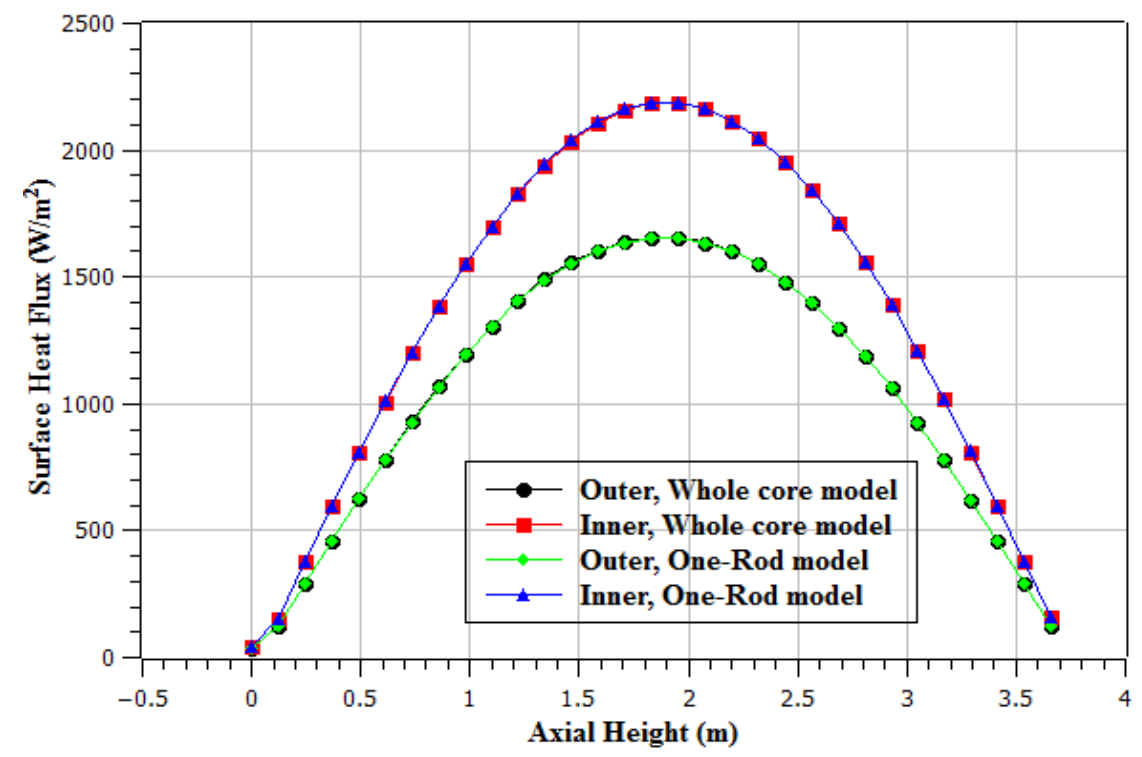

The distribution heat flux distribution and pressure drop are presented in the Figure 8 and the Figure 9, respectively. The graph of Figure 8 aims to compare the agreement between the power distributions of the models in the STHIRP. Due to the good agreement in the comparison of the models of a VIPRE rod and the STHIRP code, it is plausible then to simulate the core model for checking the STHIRP code capacity and evaluating the reactor core loaded with ring rods. 
Figure 9: Pressure drop in channels 8 and 35.

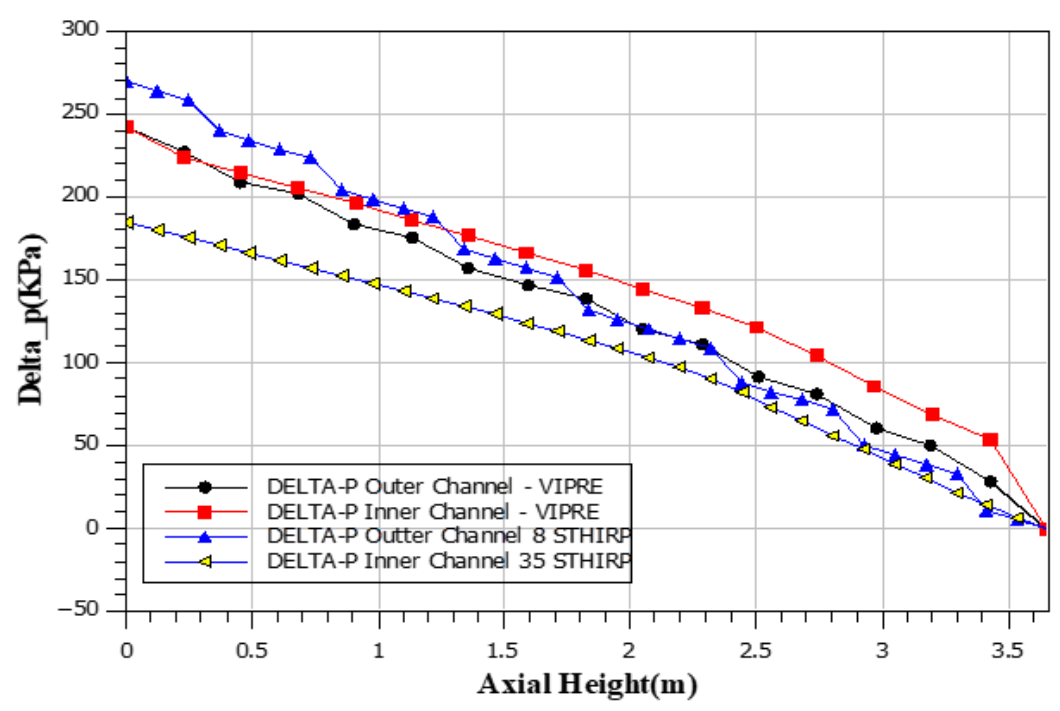

The Figure 10 shows that there is a great agreement in the DNBR behavior profile of the internal channels calculated by the codes VIPRE and STHIRP. This is one of the most important parameters of the simulation.

Figure 10: $D N B R$.

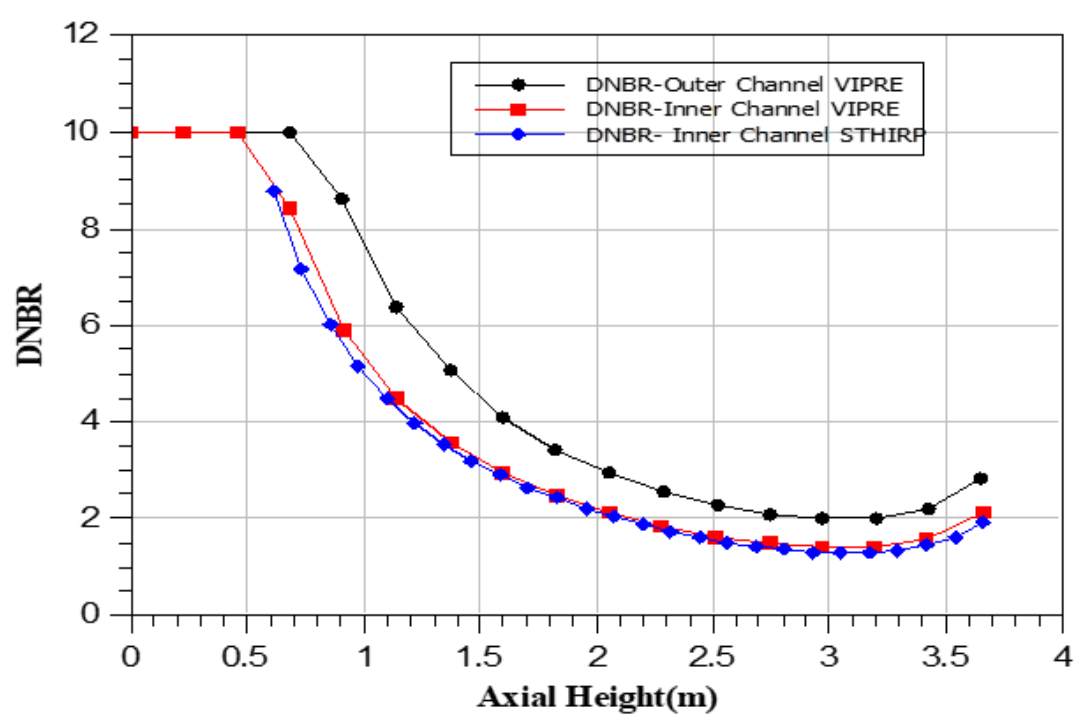

The Figure 11 shows the axial increase in temperature caused by the transfer of heat from rod 8 , shown in Figure 3, to water. 
Figure 11: Coolant temperatures in the hot inner and outer channels.

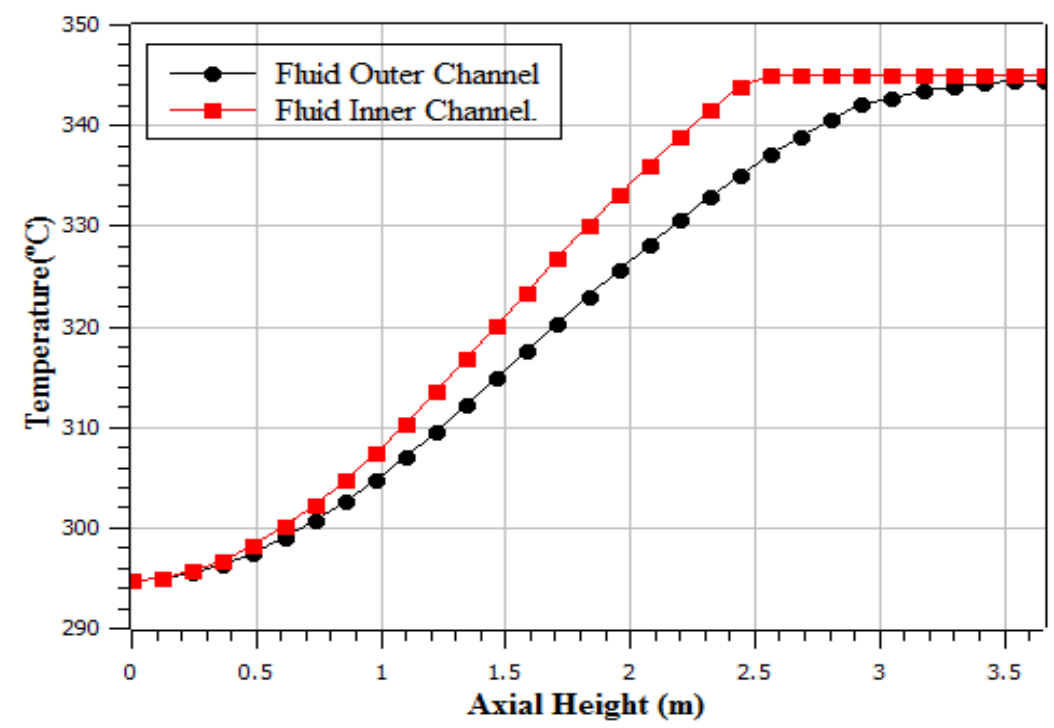

It is possible to observe the difference in temperature of the fluid when passing through the inner and outer regions of the rod 8 where the inner channel reaches saturation more quickly. The final temperature is $345.1^{\circ} \mathrm{C}$.

The Figure 12 represents the axial temperature distribution that was analysed at five different points, distributed radially according to Figure 2. The highest temperature is represented by curve $\mathrm{T} 3$, in the region central between the intern and extern claddings.

Figure 12: Axial temperature distribution in claddings.

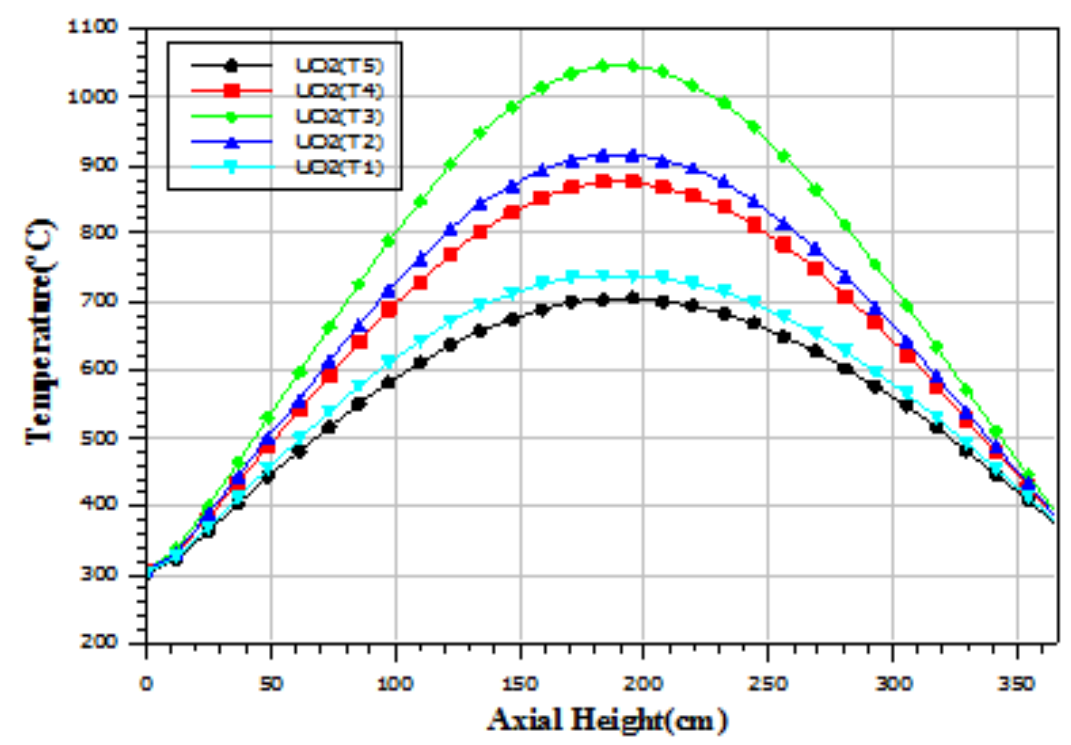


In the Figure 13 there are three checkpoints at the cladding outter and three checkpoints at the cladding inner. Checkpoint 1 is in contact with water and point 3 is in contact with the helium gas between the refrigerant and the pellet.

Figure 13: Temperature distribution in the internal and external claddings.

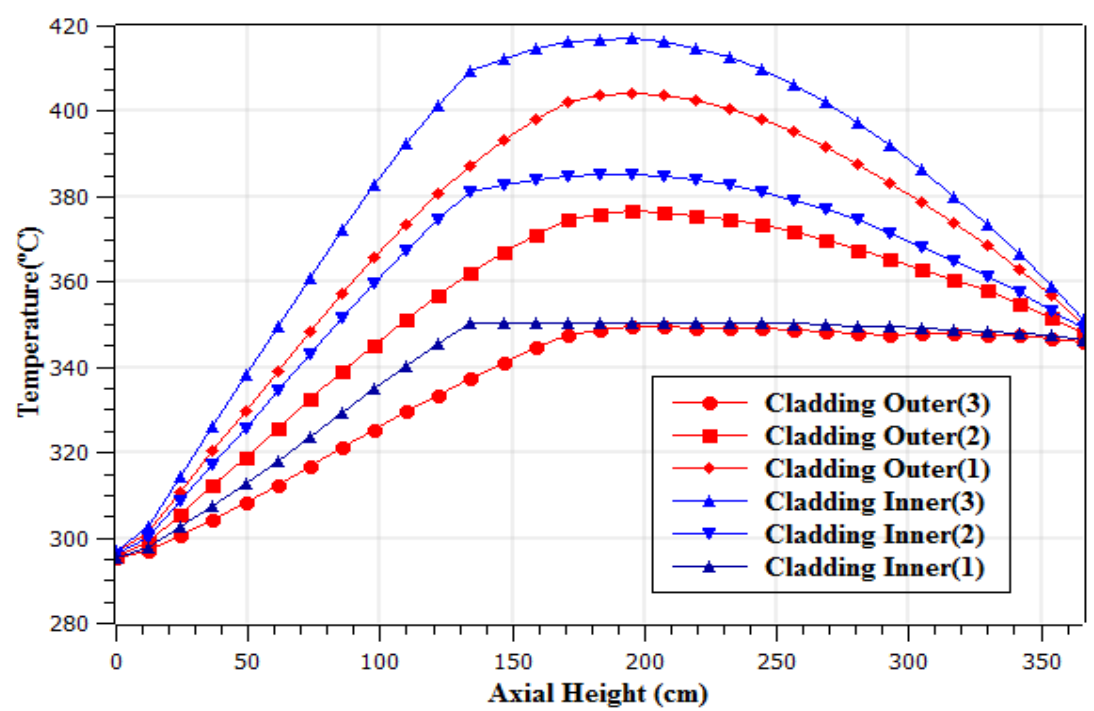

The results of the changes in the core model due to changes in thermal conductance resulting from the fuel burning are shown in the Figure 14. Burning may imply swelling, cracking, or displacements of the pellets and this parameter alters not only the temperature of the fuel but also the MDNBR. The conductance value is provided by Kazimi [6].

Figure 14: Comparison of temperature distributions

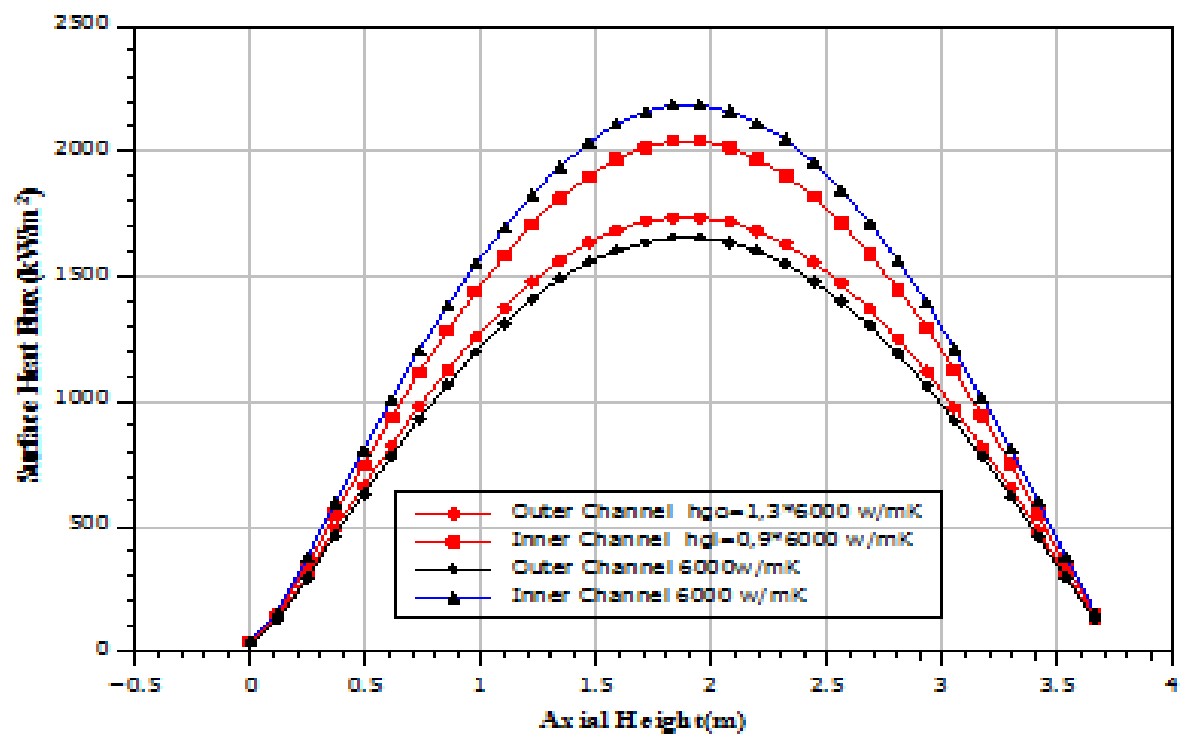


This change in the heat flux causes an increase of MDNBR that was of 1.29 which is within the security range [7] and becomes to 1.699, still occurring in the internal channel. The Figure 15 shows the new DNBR profile obtained from the proposed alteration. In this case there is an increase in safety.

Figure 15: DNBR whole core model and whole core model with change of the gap conductivity.

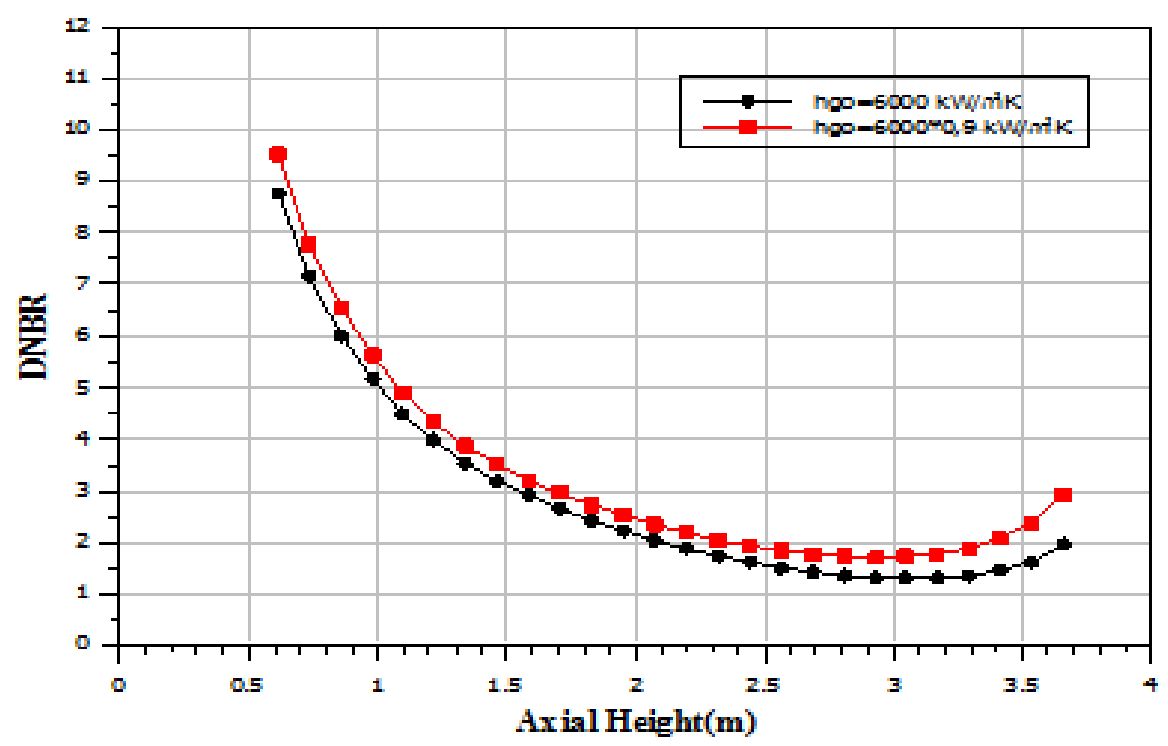

The delta-p presents a little difference between models in inner channel 35 in Figure 16. The results for extern channel number 8 have good agreement.

Figure 16: Comparison of pressure drop

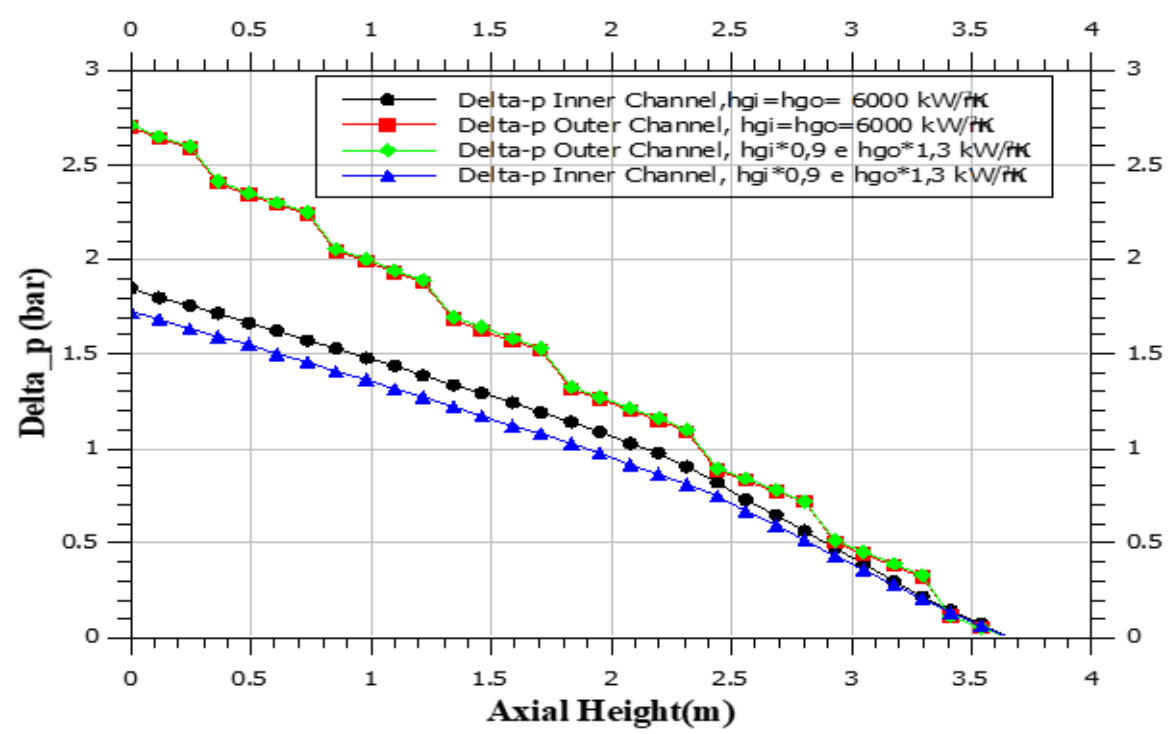


The behavior of the water temperature shown in the Figure17 presents a uniform variation between the internal and external channels.

Figure 17: Temperature distribution of the water in channels.

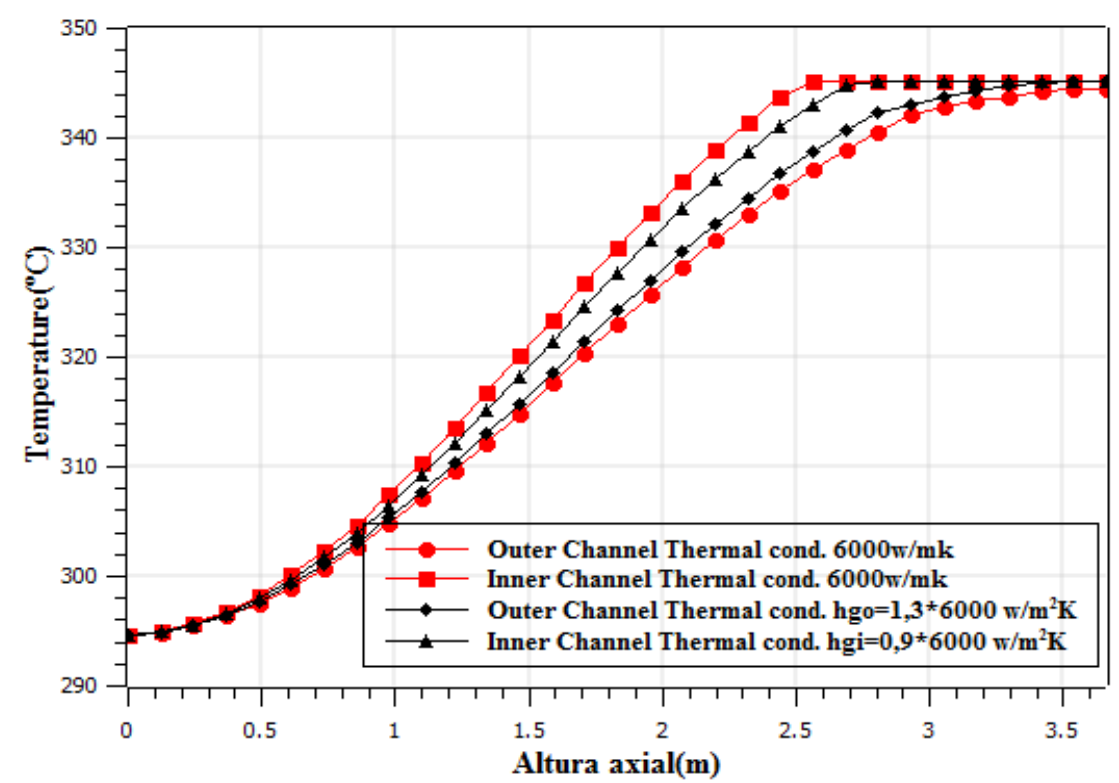

Concerning the use of annular fuel, the results presented are satisfactory considering the data available in the literature and in the reference report.

These results reinforce the expectation of using annular fuels in power reactors and even inserting them from existing reactors by replacing the common fuel element for an element with annular fuel, since the external dimension of the fuel elements has been maintained.

\section{CONCLUSION}

In order to evaluate the capacity of the STHIRP-1 program, especially about to the thermal model, the analysis of the annular fuel concept was performed. The calculations were carried out always seeking to follow the information contained in the reference report. The results showed that, in general, there is a good correspondence between those predicted by STHIRP-1 and those presented in the reference document. 
The predicted results at the model of a rod and for the core model show that the lowest value of the nucleated boiling-point limit (DNBR), remains above the minimum value of 1.30, indicating that the occurrence of critical heat flux in the reactor core will be very unlikely.

The one-rod model: Through the results of heat flow distributions it is possible to conclude that the STHIRP program has a formulation capable of split the heat flux and presents a good agreement with the values presented in the reference report. The values obtained from MDNBR are above the minimum required and with a difference of less than $5 \%$ concerning to the reference report.

The whole core model: It represents a more accurate simulation of the thermal hydraulic conditions of the reactor. In this model, the MDNBR and heat flow were obtained with excellent agreement with the reference report. The difference between the temperatures in the center of the fuel has a good safety range against the melting of the pellets. The temperature of the water saturates at approximately $345^{\circ} \mathrm{C}$, although the temperature of the channel within the fuel is higher to some extent of the channel.

Model with altered gap thermal conductivity: The simulated change results in a better balance in the pellets between the internal and external temperatures, in the distribution of the heat flux and in the temperature distribution of the fluid. The new value of the maximum internal heat flux that was $2046.5 \mathrm{~kW} / \mathrm{m}^{2}$ decreased to $2038.96 \mathrm{~kW} / \mathrm{m}^{2}$ and the external flow increased from $1654.18 \mathrm{~kW} / \mathrm{m}^{2}$ to $1736.26 \mathrm{~kW} / \mathrm{m}^{2}$. This means a reduction in the inner channel of $0.36 \%$ and an increase in the outer channel of $4.96 \%$. The value of the MDNBR also increases about $20 \%$ in the internal channel. Finally, the eventual change of thermal conductivity in the gap, if it occurs in normal operation, favors the safety and efficiency of the reactor.

The annular fuel is promising in terms of safety and efficiency. The simulation of this fuel is difficult and complex. There are few computational programs adequate for their analysis. The results obtained with STHIRP-1 prove the analytical capacity of the code in the evaluation for this fuel considered innovative without the need of any adaptation.

\section{ACKNOWLEDGMENTS}

The authors are grateful to Fapemig, CNPq, Capes and CNEN for the support by support, and at DEN (UFMG) for the structure and opportunity. 


\section{REFERENCES}

${ }^{[1]}$ Blinkov, V. N. et al. Prospects for Using Annular Fuel Elements. Thermal engeneering, Moscou Oblast, v. 57, n. 3, p. 2013-2018, 2010. ISSN 0040-6015.

${ }^{[2]}$ Silva, R. H. M. Estudo de combustível anular para $P W R$. Universidade Federal de Minas Gerais. Belo Horizonte, p. 71. 2017.

[3] Veloso, M. A. F. Análise Termofluidodinâmica de Reatores Nucleares de Pesquisa Refrigerados a Água Em Regime de Convecção Natural. Tese em Engenharia Química Sistemas de Processos Químicos e Informática-Universidade Estadual de Campinas(UNICAMP). Campinas, p. 231. 2004.

${ }^{[4]}$ Han, K. H.; Chang, S. H. Development of a thermal-hydraulic analysis code for annular fuel assemblies. Journal Nuclear Engeenering and Design, Yuseong-gu, v. 1, n. 226, p. 267-265, Julho 2003. ISSN 0029-5493.

${ }^{[5]}$ Shin, C.-H. et al. Thermal hydraulic performance assessment of dual-cooled annular nuclear fuel for OPR-1000. Nuclear Engineering and Design, Daedeok-Daero, v. 1, n. 243, p. 9, Dezembro 2012.

${ }^{[6]}$ Kazimi, M. S.; Hejzlar, P. High Performance Fuel Design for Next Generation PWR's. MITCenter for Advanced Nuclear Energy Systems. Massachusetts, p. 292. 2006.

${ }^{[7]}$ Duderstadt, J. J.; Hamilton, L. J. Nuclear Reactor Analisys. $2^{\mathrm{a}}$. ed. Michigan: Jon Wiley and sons, v. I, 1976. 